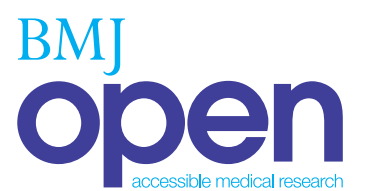

\title{
General practitioner experience and perception of Child and Adolescent Mental Health Services (CAMHS) care pathways: a multimethod research study
}

\author{
Saba Hinrichs, ${ }^{1}$ Matthew Owens, ${ }^{2}$ Valerie Dunn, ${ }^{2}$ Ian Goodyer ${ }^{2,3}$
}

To cite: Hinrichs S,

Owens M, Dunn V, et al. General practitioner experience and perception of Child and Adolescent Mental Health Services (CAMHS) care pathways: a multimethod research study. BMJ Open 2012;2:e001573.

doi:10.1136/bmjopen-2012001573

- Prepublication history and paper are available online. To view these files please visit the journal online (http://dx.doi.org/10.1136/ bmjopen-2012-001573)

Received 29 May 2012 Accepted 27 September 2012

This final article is available for use under the terms of the Creative Commons Attribution Non-Commercial 2.0 Licence; see http://bmjopen.bmj.com additional material for this

\section{ABSTRACT}

Objectives: This is a pilot study with the objective of investigating general practitioner (GP) perceptions and experiences in the referral of mentally ill and behaviourally disturbed children and adolescents.

Design: Quantitative analyses on patient databases were used to ascertain the source of referrals into Child and Adolescent Mental Health Services (CAMHS) and identify the relative contribution from GP practices. Qualitative semistructured interviews were then used to explore challenges faced by GPs in referring to CAMHS.

Setting: GPs were chosen from the five localities that deliver CAMHS within the local Trust (Peterborough City, Fenland, Huntingdon, Cambridge City and South Cambridgeshire).

Participants: For the quantitative portion, data involving 19466 separate referrals were used. Seven GPs took part in the qualitative interviews.

Results: The likelihood of a referral from GPs being rejected by CAMHS was over three times higher compared to all other referral sources combined within the Cambridge and Peterborough NHS Foundation Trust. Interviews showed that detecting the signs and symptoms of mental illness in young people is a challenge for GPs. Communication with referral agencies varies and depends on individual relationships. GPs determine whether to refer on a mixture of the presenting conditions and their perceived likelihood of acceptance by CAMHS; the criteria for the latter were poorly understood by the interviewed GPs.

Conclusions: There are longstanding structural weaknesses in the services for children and young people in general, reflected in poor multiagency cooperation at the primary care level. GP-friendly guidelines and standards are required that will aid in decision-making and help with understanding the referrals process. We look to managers of both commissioning and providing organisations, as well as future research, to drive forward the development of tools, protocols, and health service structures to help aid the recognition and treatment of mental illness in young people.

For numbered affiliations see end of article.

\section{Correspondence to}

Dr Saba Hinrichs;

s.hinrichs@cantab.net

\section{INTRODUCTION}

In the UK Child Morbidity Survey, $10 \%$ of children and young people aged 5-16 had a

\section{ARTICLE SUMMARY \\ Article focus \\ - This pilot study investigated general practitioner involvement in the referral and management of men- tally ill and behaviourally disturbed young people. \\ - Quantitative and qualitative methods were used to identify challenges faced by GPs in referring within mental health care pathways. \\ - This study is part of a larger investigation of the detection and management of child and adolescent mental health within the Collaborations for Leadership in Applied Health Research and Care for Cambridgeshire and Peterborough (CLAHRC-CP).}

Key messages

- The likelihood of a referral from GPs being rejected by Child and Adolescent Mental Health Services (CAMHS) was over 3 times higher compared to all other referral sources combined within the Cambridge and Peterborough NHS Foundation Trust (CPFT).

- Detecting the signs and symptoms of mental illness in young people is a challenge for GPs; there is no standardised specialised-knowledge base nor protocol-driven decision-making tool from which GPs can develop their clinical decision-making skills in this area.

- GPs determine whether to refer on a mixture of the presenting conditions and their perceived likelihood of acceptance by CAMHS; the criteria for which appear poorly understood by the interviewed GPS.

diagnosed mental health disorder, involving a level of distress or social impairment likely to warrant treatment. ${ }^{1}{ }^{2}$ These conditions result in substantial long-term burden to parents, ${ }^{3}$ and many result in considerable, long-term costs to society if conditions persist into adulthood. ${ }^{4}{ }^{5}$ The detection of mental disorders in the young can and should start at community and primary care levels, but national and local priorities, together with finite resources and psychological barriers to accessing services, often result in limiting the opportunities for early detection in schools 


\section{ARTICLE SUMMARY}

Strengths and limitations of this study

- The design of this study was supported by the mix of available scientific and clinical expertise and driven by contextual knowledge of the current needs and challenges of child and adolescent mental health within this geographical region.

- The questions posed to GPs were open-ended to allow themes to emerge during interviews. The findings provide valuable insights into the challenges of referring into CAMHS in this region as the GPs were exclusively chosen from the five localities therein.

- Because of limitations of sample size, organisational and geographical distributions, this study is hypothesis generating and results may not be generalisable to the GP practises in the rest of England or primary care services elsewhere. However, the conclusions confirm similar findings published in other UK and international studies.

and families. The World Health Organisation has noted that barriers to care for child and adolescent mental health in developed countries include stigma for both patients and their caregivers, priority given to illnesses labelled 'physical' without the recognition of the association with mental disorders, and the reduction of workforce in community settings. ${ }^{6}$

Care pathways are management tools for tracking the route of a well-defined (eg, diagnostic or needs-based) group of patients over a specified period of time. ${ }^{7}$ They can serve as useful frameworks for supporting care providers with evidence-based guidelines across community, primary and secondary care settings, provided they are implemented with sign-up from all professional groups. In Child and Adolescent Mental Health Services $(\mathrm{CAMH})$, these pathways often involve service providers that range across clinical, social educational and third care sectors. Although international ${ }^{6} \quad 8 \quad 9$ and UK national $^{10}{ }^{11}$ publications exist for guiding the design of these pathways, they are also produced at the local level. ${ }^{12}$ Previous work on CAMHS, however, suggests that there are challenges both in implementing evidencebased guidelines ${ }^{13}$ and in designing and delivering care pathways, ${ }^{14}{ }^{15}$ as well as challenges in their effective implementation. ${ }^{16} 17$

The National Health Service (NHS) is the publicly funded healthcare system in the UK and is free at the point of use for anyone who is resident in the UK. Operating within primary care within the NHS, the general practitioner (GP) acts as a gatekeeper for many services in secondary care (acute and specialised health care). Care pathways in mental health services in the UK span across primary and secondary care. Therefore, the GP may be a part of the pathway itself, as he/she may be consulted after referral, and during-or-after care is delivered along the pathway. However, the factors that influence the success rates of GP referrals into CAMHS, and the efficient and effective use of a care pathway (including the role of the GP through the mental-health treatment process), remain unknown. It should be noted that referral by a GP does not guarantee that the patient will be seen by specialist services. GPs are not expected to make a diagnosis before referring to specialists either; referrals can even be made for diagnostic purposes given a sufficient level of dysfunction.

Care pathway studies in the UK have focused to date on the ability of health professionals, including GPs, to detect and recognise child mental health problems in primary care settings. In a study in South London, 5 general practitioners assessed 186 children whose parents also completed a standard screening questionnaire for the recognition of psychiatric disorders. ${ }^{18}$ On examination of the child, GPs detected no more than one in three cases who met parent-rated criteria for a psychiatric disorder. ${ }^{18}$ The expression of parental concern to the GP substantially increased recognition of four of five such cases. Reviewing the pathways to care literature, Sayal ${ }^{19}$ concluded that parental concerns were key moderators for aiding GP recognition of mental health problems in their children.

Identifying barriers to recognition has been relatively successful and resulted in identifying quality standards that could be implemented in primary care settings. ${ }^{20}$ Key factors in facilitating seeking help for troubled children are longer appointment times, trusting relationship and continuity of care with the GP. ${ }^{21}$ The study noted barriers for parents seeking help included embarrassment, stigma of mental health problems including diagnosis, being judged a poor parent and their child being removed from the family should they seek help.

These important studies demonstrate characteristics that can be managed to improve recognition of troubled children by GPs and facilitate entry into the care pathway system. To date, however, there has been little investigation of GP perception and experience of referral into the child care pathway system once a mental health or behavioural problem in a child or adolescent has been duly recognised.

The aim of the research in this article was to investigate for the first time whether GP experience and perception of the CAMHS care pathway influenced their decision-making process to refer cases for further specialist assessment. The study was undertaken within the Cambridge and Peterborough NHS Foundation Trust (CPFT) which has a monopoly provision for moderate-to-severely mentally ill patients under 18 for the County of Cambridgeshire, including the Independent Local Authority of Peterborough, covering a total population of approximately 700000 individuals and around 170000 under 19 years of age. ${ }^{22}$ GP services refer to outpatient clinics situated in five localities across the county (Peterborough City, Fenland, Huntingdon, Cambridge City and South Cambridgeshire) and have access to three inpatient units for young people (eatingdisorders specialist unit, adolescent-inpatient, and children-inpatient mental illness units) all situated on one site in South Cambridgeshire. The investigation used both quantitative and qualitative methods to 
identify challenges faced by GPs in referring within mental health care pathways.

This pilot study was carried out as a prelude to designing a larger investigation of GP involvement in the management of child and adolescent mental health within the Collaborations for Leadership in Applied Health Research and Care for Cambridgeshire and Peterborough (CLAHRC-CP). CLARHC-CP is 1 of 9 programmes funded by the National Institute of Health Research (NIHR) in 2008 to undertake high quality applied health research focused on the needs of patients and service users, and to support the translation of research evidence into practice in the NHS and social care. CLAHRC-CP focuses on people with mental illness, intellectual (learning) disabilities, acquired brain injury, and care for those approaching the end of life. This project is one of many steps for consideration in a broader transformation of CAMHS. Working within the CLAHRC alongside providers from the local mental health hospital was useful for providing contextual knowledge of the general challenges of addressing mental health issues for young people within the area. Furthermore, the CLAHRC-CP project provided the opportunity to bring together researchers from different disciplines, including psychiatry, clinical psychology, health services research and process engineering design. The design of this study was therefore supported by this mix of available expertise, and driven by contextual knowledge of the current needs and challenges of child and adolescent mental health within this geographical region.

\section{METHODS}

We initially adopted a quantitative approach to analyse the CPFT patient database to ascertain the source of referrals into CAMHS with a particular focus on the relative contribution from GP practices. Data were available from the period including April 2006 to March 2011 involving 19466 separate referrals, and we calculated the percentage of referrals by source. Logistic regression models were then specified to estimate the likelihood of a GP referral being rejected by CAMHS relative to all other sources combined (see table 1). Referral source categories were collapsed so that $95 \%$ of referrals were given specific categories, with the remainder classified as 'Other Source of Referral'. ORs are reported, along with associated $\mathrm{p}$ values and $95 \%$ CIs.

Subsequently, qualitative methods based on face-to-face interviews with selected GPs were then used to obtain deeper insight into the perceptions and experiences in the decision-making processes when referring to CAMHS. These findings provide an analysis of the reasons for GPs' behaviour in relation to external drivers and regulations, as well as their views on the present conditions in the service. The first part of the interviews were semi-structured, to allow for free discussion around the concepts provided by the interviewer, ${ }^{23}$ and hence explore the experiences of GPs in relation to CAMHS and their understanding and experience of the services available to them. In the second half of the interview, the interviewer used diagrams as part of the procedure to stimulate further discussions and to prompt further elaboration on their understanding of the referral process. The diagrams served as elicitation tools ${ }^{24}$ during the interview process and were therefore also analysed together with the responses from the interview questions. These methods were based on approaches tried in previous healthcare studies that draw on engineering and modelling methods. ${ }^{24}{ }^{25}$ A copy of the format of the semistructured interview and diagrams used are included in the Appendix. The interviews lasted between 50-60 min and were held at the GP practice. Verbatim quotes directly from the interviewees' responses are shown in the boxes in the results in double quotation marks. Interviews were recorded and transcribed, and analysed using thematic coding which emerged primarily from the data itself. ${ }^{26}$ The full analysis of the themes that emerged from the study, however, was conducted in conjunction with insights gained from key informants within the CAMHS, as well as GP tutors who were consulted before and after the interviews were completed.

GPs were chosen from the five localities that deliver CAMHS within the Trust (Peterborough City, Fenland, Huntingdon, Cambridge City and South Cambridgeshire) and GP practices were identified through the Postgraduate Medical Centre at the University of Cambridge Clinical School, as well as the Primary Care Research Network (PCRN). They were chosen to include geographical spread (2-3 GPs per each of the four areas), and varying social demographics of the patient population. No other exclusion criteria were used. A total of 19 GPs plus 2 GP practice managers were contacted by email. Including the GP practices, at least eight of the targeted GPs were from rural areas and ten from urban areas. Ten of the directly contacted GPs were male and seven were female.

Nine GPs agreed to take part and seven were interviewed; either no responses were received from the rest or they were unwilling to participate with no reason given. Of those interviewed, four were from urban areas, three from rural, three were male and four were female. Their years of experiences working as a GP varied from 6 to 31 years. We therefore observed a balance of social demographics and gender both in the overall targeted GPs and those who agreed to take part. Given the low response rate, interviews were continually analysed as further interviewees were sought, in order to assess the consistency and variability in the perceptions expressed by the GPs. The themes formed and conclusions made are therefore only those that reflect perceptions and theme patterns observed across all the GPs interviewed. Following communication with the Cambridge branch of the NHS National Research Ethics Service, the authors were informed that this pilot study is classed as a service evaluation of professionals and therefore did not require formal ethical review. 
Table 1 Sources of referral into local (CPFT) CAMHS

\begin{tabular}{|c|c|c|c|}
\hline Source of referral & Frequency & Percentage of total & Cumulative percentage \\
\hline GP referral & 8082 & 41.52 & 41.52 \\
\hline Other source of referral & 2625 & 13.49 & 55.00 \\
\hline Consultant other than $A \& E^{\star}$ (acute) & 1777 & 9.13 & 64.13 \\
\hline Internal & 1434 & 7.37 & 71.50 \\
\hline Local authority social services & 1412 & 7.25 & 78.75 \\
\hline Education establishment/services & 1295 & 6.65 & 85.41 \\
\hline Other clinical speciality (mental health) & 1,029 & 5.29 & 90.69 \\
\hline $\mathrm{A}+\mathrm{E}$ department (mental health) & 478 & 2.46 & 93.15 \\
\hline Self-referral & 271 & 1.39 & 94.54 \\
\hline Other-referred to consultant (acute) & 185 & 0.95 & 95.49 \\
\hline Not known & 135 & 0.69 & 96.18 \\
\hline Allied health professional (AHP) & 109 & 0.56 & 96.74 \\
\hline Specialist nurses or AHPs & 108 & 0.55 & 97.30 \\
\hline Legal courts (mental health) & 96 & 0.49 & 97.79 \\
\hline Consultant referral request & 95 & 0.49 & 98.28 \\
\hline A \& E referral (acute) & 87 & 0.45 & 98.73 \\
\hline Specialist nurse (secondary care) & 70 & 0.36 & 99.09 \\
\hline Following emergency admission & 60 & 0.31 & 99.39 \\
\hline Consultant for the trust & 32 & 0.16 & 99.56 \\
\hline Emergency services $(A+E)$ & 26 & 0.13 & 99.69 \\
\hline Following $A+E$ attendance (cancer) & 22 & 0.11 & 99.80 \\
\hline Probation services (mental health) & 7 & 0.04 & 99.84 \\
\hline Carer (mental health) & 6 & 0.03 & 99.87 \\
\hline Same consultant & 5 & 0.03 & 99.90 \\
\hline Police & 3 & 0.02 & 99.91 \\
\hline GPs with special interest & 2 & 0.01 & 99.92 \\
\hline General dental practitioner & 2 & 0.01 & 99.93 \\
\hline High security (mental health) & 2 & 0.01 & 99.94 \\
\hline Optometrist & 2 & 0.01 & 99.95 \\
\hline Permanent transfer from mental health unit & 2 & 0.01 & 99.96 \\
\hline 'Choose and book' $†$ & 1 & 0.01 & 99.97 \\
\hline Consultant referral (cancer) & 1 & 0.01 & 99.97 \\
\hline Health Care Professional (HCP) A+E & 1 & 0.01 & 99.98 \\
\hline Medium security (mental health) & 1 & 0.01 & 99.98 \\
\hline Minor injuries units & 1 & 0.01 & 99.99 \\
\hline National screening Programmes & 1 & 0.01 & 99.99 \\
\hline Optometrists and orthoptists & 1 & 0.01 & \\
\hline Total & 19466 & 100.00 & \\
\hline
\end{tabular}

The categories of referral were collapsed retaining those for the first 95\% (bold) and collapsing the remainder (grey) into a single 'other' category for figure 1 .

${ }^{*}$ Accident and emergency hospital.

†NHS electronic patient self-booking system.

CPFT, Cambridge and Peterborough NHS Foundation Trust; CAMHS, Child and Adolescent Mental Health Service.

\section{RESULTS}

Referral patterns for the region

Over the 5 year research period there were 19,466 referrals in total (males $=10769,55 \%$; females $=8697,45 \%$ ). Of these, $5865(30 \%)$ were classed as multiple cases as they had been referred on two or more occasions. The distribution of all referrals by referring agency is given in table 1 and was subsequently summarised for inferential analyses. CPFT data showed that referrals came from a wide range of sources including professionals working in the NHS as well as Education, Social Services and the Probation Service (see table 1). The vast majority of referrals $(95 \%)$ were attributed by the Trust as coming from: GPs, other sources, consultants, internal sources, the Local Authority, education, mental health specialists, accident and emergency hospitals, and self-referrals. GP referrals accounted for $8082(41.5 \%)$ of the referrals making them by far the largest single referral source.

The likelihood of a GP referral being rejected by CAMHS was over three times higher than all other sources combined (see figure 1; OR=3.27, $\mathrm{p}<0.001,95 \%$ CI 2.95 to 3.63). This rejection incidence showed a significant sex by referral source interaction $(\mathrm{OR}=.80$, $\mathrm{p}$ $<0.05,95 \%$ CI .64 to .99) being slightly higher for boys $(\mathrm{OR}=3.67, \mathrm{p}<0.001,95 \%$ CI 3.21 to 4.19$)$ than girls $(\mathrm{OR}=2.92, \mathrm{p}<0.001,95 \%$ CI 2.47 to 3.45$)$. The CPFT data also showed that almost 6000 of these were multiple referrals, which may suggest particular challenges in 


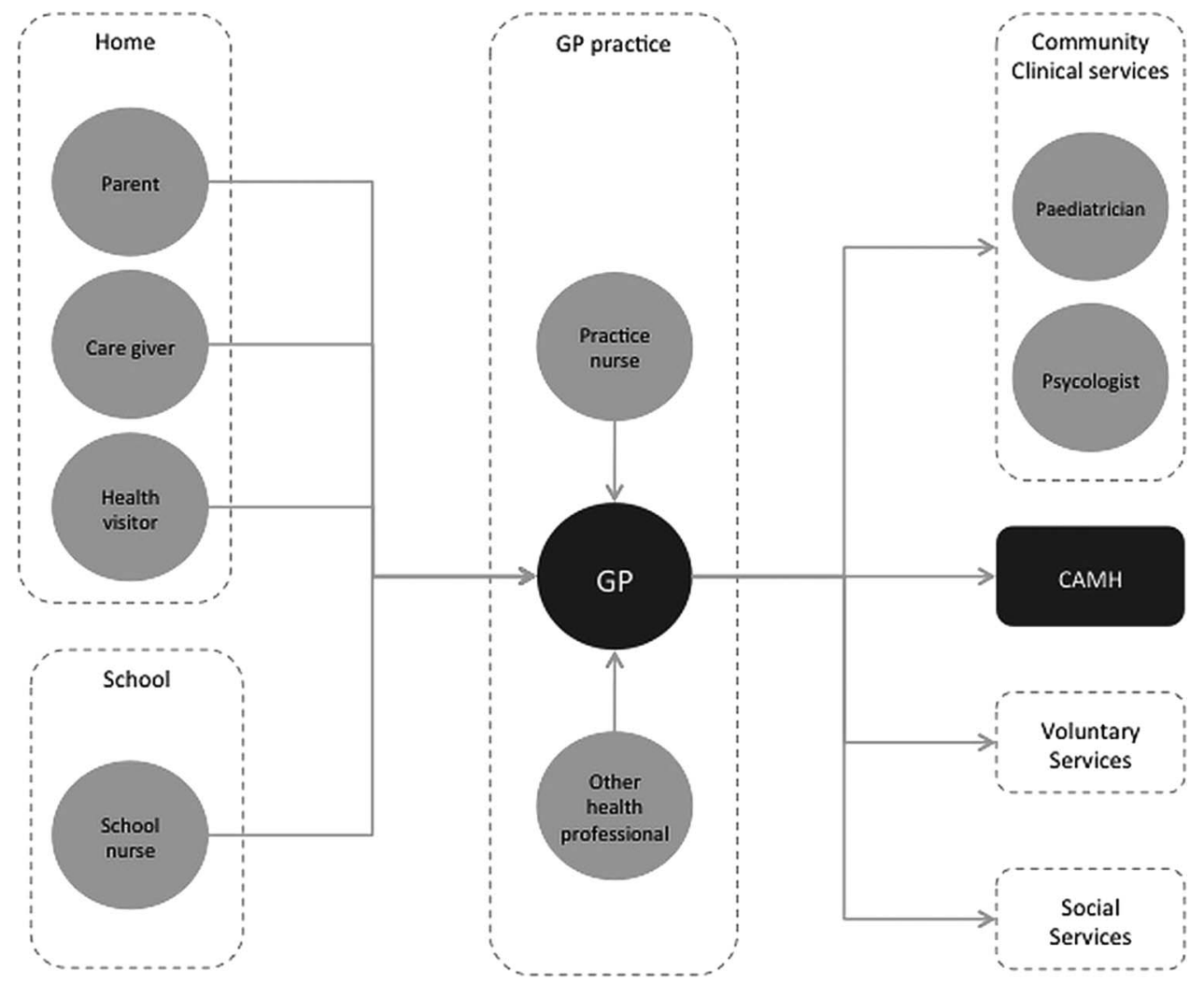

Figure 1 Examples of general referral routes available, as drawn by the general practitioners interviewed.

accessibility and/or diagnosis. We therefore further checked whether the higher incidence of rejection was not a product of this process by further analysing the data using those referred once only $(n=13601)$. This analysis still revealed a similar association between GP referral source and rejection status $(\mathrm{OR}=3.23, \mathrm{p}<0.001$, $95 \%$ CI 2.84 to 3.67 ). Within the observation period of the study there was no GP referral source $\mathrm{x}$ single referral interaction $(\mathrm{OR}=0.90, \mathrm{p}=.333,95 \%$ CI 0.72 to 1.12$)$. The odds risk ratio for likelihood of rejection for single referrals was therefore no different when compared to multiple referral cases $(\mathrm{OR}=3.60, \mathrm{p}<0.001,95 \%$ CI 3.01 to 4.32 ), within the observation period.

The precise reasons for this threefold increase in rejection of GP first time referrals, compared to all other sources of referral, cannot be determined from this quantitative analysis. GPs may provide insufficient information in their referral letter, make referrals that are outside the current acceptance criteria of moderate-to-severe mental illness or behavioural disturbance up to and including 17 years of age, or be unaware of alternative or better resources for the patient's particular problems. The challenge of this decision-making process in making a referral is therefore the key element investigated in the qualitative study.

\section{Challenges in the referral process}

The second phase of this study was to utilise qualitative methods to investigate the challenges that may contribute to high rejection rates of referrals from GPs to CAMHS, by capturing the perceptions and experiences of GPs in the referral decision-making process. The themes from interviews were grouped into 3 sections: (1) identifying mental health symptoms, (2) choosing whether to refer and (3) communicating with referral agencies.

\section{Identifying mental health symptoms}

The qualitative findings show that, among the GPs, there are heterogeneous concepts and precepts regarding the nature of mental illness. In the International Classification of Diseases (ICD 10), a mental health diagnosis is achieved by assessing a clinically recognisable set of signs and symptoms associated with personal functional impairment. ${ }^{27}$ The GPs interviewed reported difficulties in the recognition and detection of mental health signs and symptoms in young people. From a GP's perspective, a child or adolescent mental illness could be seen as clinically, or socially, defined. Such perspectives were clear from the comments made to the researcher (box 1). Whereas in the case of adult mental health, GPs have at their disposal a checklist that helps decide on the need for a referral, no such mental health tool was known to exist for UK GPs to use with children and adolescents.

Interestingly, all seven GPs interviewed reported that they seldom see emotional and behavioural problems in the under-18-year-old population (ranging from one to up to eight referrals a year per GP). Furthermore, when 
Box 1 Selected comments from general practitioner in identifying mental health symptoms

"On the whole, the real mental health issue is very very low to be honest... it's more behavioural and more social..." (GP6)

"(I see) children who are very stressed and are having lots of difficulties, not mental health difficulties necessarily but emotionally, which is probably more of a social work issue, but yeah, it also gets into mental health." (GP 3)

"Where is psychiatry stopping and where do we get into educational psychology? Where are we going into mild behavioural problems, and how much is it parents not helping?" (GP1)

"The alcohol thing is not about mental health, it's about education!" (GP6)

"We don't have that much full on craziness here (in this village)..." (GP5)

asked whether they remembered a case when a family or teenager came in for one complaint but other symptoms were noticed that were indicative of mental health, all admitted to never having had such a case. Yet, so-called atypical or masked presentations are relatively common for psychiatric disorders in adults. ${ }^{28}$ There may be a reluctance to undertake mental health assessment if a younger patient or their parent comes in with another complaint (eg, a sore throat) as suggested by one GP who considered that, in this case, asking deeper questions would be 'inappropriate' (GP3).

Many of the comments reflect a lack of consensus between GPs in evaluating complex presentations of mental illness. One GP commented that it was easier to detect children as they are 'pretty straightforward' (GP3), since their problems are mostly behavioural problems, although another stated 'they don't come to light so easily... it's usually some kind of behavioural problem... they don't come and tell you' (GP6). Although there are standardised diagnostic criteria for detecting clinical syndromes that involve antisocial behaviour, ${ }^{27}$ GPs differed markedly in their understanding of these. A similar observation was noted for mental illnesses that present with mood changes. While one GP commented on how body language can give the signal 'I'm miserable' (GP4), and therefore needs some consultation to find out the source of the issues, another commented that they would not refer a 'slightly gloomy and grumpy teenager' (GP1). In fact, irritability occurring most days, showing little diurnal variation and lasting more than 2 weeks, and out of context and culture for that teenager, may be a mood signal for unipolar depression. ${ }^{29}{ }^{30}$ This same GP stated he would not refer if the teenager presented 'a little bit of cutting just to enjoy ourselves... that could just be a way of getting attention!' (GP1). Recent studies have shown that nonsuicidal self-injury (NSSI) is indeed relatively common, occurring in some $10-15 \%$ or so teenagers. ${ }^{31}$ Furthermore, NSSI is associated with a 5-10-fold increase in suicidal thoughts and behaviours in depressed teenagers. ${ }^{32}$ These recent research findings highlight the key importance and responsibilities of CAMHS research into practise communications to GPs when new facts of high clinical relevance are published.

\section{Choosing whether to refer}

Even when mental health symptoms have been identified, a second group of challenges exist in deciding when and whether to refer. All of the GPs interviewed stated they refer when there are significant overt mental illness risks, such as physical signs of recurrent self-harm and report of suicidal attempts. However, even among this small sample of interviewed GPs, there was significant variation in the other, subtler, types of triggers that would warrant a referral. These themes were mainly around more generic 'risky' issues, within the following domains:

- Evidence of child abuse (GP1, GP3)

- Need for medication/therapy that I cannot prescribe (GP1, GP2)

- Huge temper/anger management signs (GP 3, GP 6)

- Panic and anxiety symptoms (GP 2)

- ADHD symptoms (GP 2)

- If child had been in local authority care (GP 2)

- Behaviour problems (GP 1, GP2, GP3)

A referral was also made if the GP felt they needed to prescribe medication, access to further therapy, and a further diagnosis or specialists' opinion. The selected comments in box 2 show how the GPs interviewed all expressed the need for specialist knowledge for detecting, treating and managing mental health disorders in young people, and that this knowledge is not necessarily one that they possess. Despite this overall recognition of the importance of specialist knowledge of others in the care pathway, each GP had a differing view on their own capability in delivering treatment for mentally ill young people. Of the seven interviewed GPs, only two reported having prescribed antidepressants to young people or children, but the other five said they would not, under the current guidance from NICE. ${ }^{29}$ Only one of the GPs felt comfortable to see the patient again and try methods such as cognitive behavioural therapy (CBT) and prescribe medication if necessary (box 2). A key part of the lack of referral even for a full assessment was because of the time restrictions on GP appointments in giving justified time required for a diagnosis and formulation (box 2). In some GP practices, there are monthly multidisciplinary meetings with specialist agencies that are child and family focused, at which school nurses and social services may participate, which may buffer the non-specialist knowledge base of the medical practitioner.

The findings suggested clearly that an overt risk factor is never ignored as seen in the second comment in box 2 . However, it seems that the decision to refer is not always clearcut, nor dependent solely on the GP's judgement. As with many other conditions, this does also depend on what is likely to be acceptable to the parent (one GP 
Box 2 Selected comments from general practitioner in choosing whether to refer

"I will get the child to come back and see me another 2-3 times before I decide to refer" (GP4)

"I think the culture among GPs now is to self-manage and not refer because even if you do refer it does take a while but if they are harming themselves, then Yes (I would refer)" (GP6)

"I find no other area better than psychiatry where (psychiatric) nurses play a very big role, in the ward and in the community... but we don't have that for children" (GP6)

"Health visitors are usually on the ball. They are quite good at child protection I think that is their expertise..." (GP6)

"I am not skilled to do any necessary testing or treatment" (GP2)

"The person understands which box to put them in best, is the person who runs the service, not the person who is referring into the service... I wouldn't think of myself as a specialist in the field, I would have to refer them" (GP1)

"Specialists can do CBT, advise on managing the patient, management, prescription, counselling, CBT, family therapy... I am looking for them to assist the patients, however it is appropriate, and then treat them." (GP2)

"I don't have a proper screening tool for a child... I can't really offer anything... we can't do counselling as GPs see them for a short time... so to get any counselling needs a psychiatric referral." (GP7)

"In 10 minutes you just physically cannot provide that service, it's not something you can do, and I mean we're not the specialisation for it." (GP3)

commented on often having 'a lot of pressure from parents to refer' GP2), as well as whether the service will accept the referral. A few GPs noted that estimating the likely success of their referral is part of the tricky process they need to go through. The paperwork and process can take a while and GPs do not wish to raise the patients' or families' expectations when it is likely to be an unsuccessful referral. One GP even called the CAMH referral criteria a 'mystery'. Another GP stated that she would not refer if 'it's unlikely CAMH will take them' (GP3).

It can be concluded that the decision to refer very much depends on the overt nature of the presenting clinical conditions (ie, the risk of harm, signs of inability to function normally, parents' influence). However, these clinical decisions appear mediated by the GPs' judgement regarding the likelihood of acceptance from CAMHS. The implication is that the entry criteria for CAMHS are simply not being delivered in a clear and concise way to GP services, and that CAMHS has to consider the value that may accrue to GP both from a specialist consultation as well as a referral for potential treatment.

\section{Communicating with referral agencies}

Once the decision for referral has been made, the next step for a GP is determining the most appropriate referral route. Where they are sent very much depends on the presenting conditions. Figure 1 shows, collectively, what the GPs either drew or stated as being their referral routes. The figure only represents the possible routes taken in the referral decision-making process. This includes those agencies that refer to the GP, and those the GP refers onwards. The information collected and represented in figure 1 shows the agencies which the GPs themselves have provided in the interviews about their possible, and/or previously used, referral routes.

The GPs unanimously stated that no feedback is ever received from voluntary or social services despite this information being very important in future and ongoing diagnosis and treatment (see example comments in box 3), due mostly to their different confidentiality requirements. For many of the GPs, a good relationship was established with their local CAMHS, especially if they had access to a voice on the phone to help them with a referral query. Having only one referral route for all mental health services was also seen favourably, although retaining the option to consult other available expertise was also apparent from the interviews. Alternative expertise, however, is limited, given that liaison psychiatry nurses, commonplace for adults, do not exist for children in the UK. Despite the overall satisfaction with CAMHS, there were comments made about their 'non-communicative style' (GP2), especially in terms of their criteria and thresholds for accepting referrals. Incidentally, the key informants at CAMHS during this study noted a similar challenge in reaching GPs to participate in care management and the care pathway development process, but the reasons for these challenges have not been investigated for this region.

Finally, when presented with the latest care pathways developed locally by the Trust, only two out of the seven GPs recognised the document (it is unknown whether or not they all received it previously), and one of them called it 'nonsense'. The care pathway measure called the Child Global Assessment Scale, indexing the level of overall personal impairment of the patient while unwell and used for every CAMHS assessment, was a particular source of difficulty. None of the GPs interviewed was familiar with this scale despite its prominence in the CAMHS document. It should be noted, however, that this document may not have been intended for primary care workers, although it contains information which could be used both for education and communication of the referral process.

\section{DISCUSSION}

The quantitative analysis of the CPFT data showed that referrals originating from GPs were three times more likely to end in a rejection by CAMHS. Beginning to understand the potentially problematic referral process in more depth was achieved by a detailed qualitative inquiry with a sample of local GPs. Our information was obtained under three themes: detection of mental health symptoms, referral choices and communication with referral agents. 
Box 3 Selected comments from GPs on communicating with referral agencies

\section{Relationship with other agencies}

"(I have) very little contact with schools, not saying never.... I can’t think I can recall any contact with voluntary organisations... perhaps occasionally..." (GP1)

"I didn’t hear back from voluntary organisation. I never hear back." (GP7)

"We do not have a good relationship with social workers, and our entire relationship with social work historically has been bad because they're all changing such that they are directors to service rather than providers of services, the problems is there is no service... not in this village or area". (GP3)

\section{Relationship with Child and Adolescent Mental Health Services (CAMHS)}

"I think if you have too many (services) then it becomes too complicated... you need to try and minimise the number of people involved because I think it shows a range of emotional difficulties then I think it would probably be one thing which is mental health" (GP2)

"(I have) one CAMH referral point... but I could refer to in-house counsellors or private counsellors... (even if range of difficulties)" (GP4)

"It's nice to have a simple one referral route... it's always slightly frustrating to me to have to do child psychiatry, adult psychiatry, old psychiatry-you could say why not just have psychiatry and then we'll have a look at the problem and the age ..." (GP1)

"I don't know what their thresholds are. I mean... I think beyond CAMH you feel a bit lost actually. If they don't meet the criteria they will be bounced back and say they don't meet the criteria." (GP2)

"CAMH—-seems to have changed its criteria over time..." (GP3)

"(Our relationship) is quite good. I mean certain under pressure... and they are doing their best and I think that certainly the parameters over the years have changed and that is because they have been told to change but you know, fair enough..." (GP3)

\section{Relationship with other liaison staff}

"(Liaison psychiatry nurse for adults) is like a triage person. He takes history on the phone and then decides if this person is more suitable for counsellor or CBT ... he gives us access to all these other services-that I find very useful for the adults. I can't see why they can't do that for children!" (GP6)

"Health visitors we find very good. They have fantastic information they say I know this person that parents is always drinking, etc. She would always have knowledge, and when we are trying to keep an eye, she can go in and say weigh the children... She can get a lot of information from there which would be easier than us calling the patients to come up because the parents won't bring them to see us... I think a well- trained health visitor makes a huge difference...”. (GP6)

The first challenge noted was detecting the signs and symptoms of mental illness in young people. This is consistent with prior research outlined in the introduction noting the importance of parental concerns moderating GP sensitivity towards a troubled child. In adolescence, detection may need to be made without parents and require consideration of physical symptoms, frequent health consultations and days off school, potentially indexing a depressive disorder. ${ }^{33}$ Although some progress is being made to aid GPs in recognising and managing depressed teenagers, ${ }^{34}$ there appears to be no standardised tool available to GPs to assist them in their clinical decision-making skills. The seven GPs were unanimous in commenting on the need for specialist knowledge from the moment the patient enters their consulting room to aid their evaluation of the needs. The hypothesis generated is that the absence of management tools and structure to aid GPs in their mental health assessment, and determining the quantifiable level of risk for the young patient, is a causal factor in the higher rejection rate by CAMHS further down the care pathway. Recent findings from studies of general practitioners and primary care staff suggests that undergraduate training alone may not be enough to equip primary care professionals for mental health assessments in the community at large. ${ }^{34-37}$ If the current findings presented here are validated on a larger sample of GPs, then mental health Trusts such as CPFT will need to invest in providing clinical tools such as protocols, checklists and advice sheets and training manuals to act as key aids for clinical decision-making and care pathway choice. Recent evidence suggests both written and computerised tools are becoming available for these purposes. $^{34} 35$

Within this challenge, GP perceptions about the respective functions and responsibilities of NHS CAMHS and social care services were noted. There was marked individual variation and a lack of clarity in GP perceptions whether and where to refer with the symptoms they observed. GPs also showed marked differences in their language use regarding the concept of 'difficult' children and parents. Whether this is reflected in referral practice is not clear, but the possibility of stigmatising the difficult child as a social or even a moral deficit, rather than searching for a putative mental health problem, is suggested. This area requires much further research before any definitive conclusions can be made. The hypothesis generated here is that low knowledge levels in GPs about mental illness in children and adolescents is a driver for poor decision-making for the patient. This highlights the need for continuing professional education for GPs in this area. Indeed, recent studies in Canada have identified gaps in primary care physicians' skills as a barrier to service provision in child and adolescent mental health. ${ }^{38}$ The findings also suggest that, from a GP's perspective, the responsibility for this knowledge provision currently lies with the provider Trust, and perhaps even with CAMHS employees. It is not clear at this time that managers within NHS organisations who commission or provide CAMHS are prepared to own this knowledge responsibility as GPs might wish them to do. Furthermore, service users may still be expecting primary care to provide more of the support required. For instance, a recent study on 
psychosis early intervention services for young people has shown that service users expect GPs to advocate for and navigate the health system. ${ }^{39}$ Indeed, one could argue for a more bottom-up framework for guiding, supporting and helping primary care workers to make clinical decisions and maintain a more active and open care pathway process. The perceptions and values of managers in these organisations towards their CAMH services and their communicative stance with GPs should be a key focus for further management science research. There is also no universal strategy for health maintenance visits within the UK children's services. In adults with mental health, there is an accepted notion of 'watchful waiting' on the part of primary care services. This is less prevalent for children and adolescents as is the notion of early detection for common mental illness in the school-age child and adolescents. Developing such strategies would aid early detection and enable a more systematic use of available resources.

The second noted challenge was in choosing whether or not to refer at all, once a mental health disorder has been detected. The interviewed GPs alluded to referring when there is major risk of harm, be it from the patients themselves through suicidal attempts, or external factors such as unsafe family environments. It was noted that perhaps not all patients are really captured, as many of those who turn up at the GP practice already desire a referral, as the parents will have made the appointment for this purpose. The criteria for whether or not CAMHS will accept the referral are not clear to all interviewed GPs, unless they happen to have a good working relationship with their CAMHS representative. Having the GPs clinical decisions influenced by their knowledge of how local CAMHS will respond is of interest. This notion is neither new nor confined to CAMHS; for example, a study in France shows that $87 \%$ of GPs found patients with mental health problems difficult to refer, ${ }^{40}$ whereas in the Netherlands, it was found that GPs recognised depression less often when cases presented with fewer symptoms. ${ }^{41}$ Underdiagnosis for dementia was also reported for primary care in the UK. ${ }^{42}$ While the extent to which such information is important in decisionmaking is unclear, it demonstrates that these decisions are mediated by the GPs' understanding of, and relationship with, local services and functions, and their clinical knowledge. This is a researchable issue, which, if supported by further evidence, would further indicate the importance of CAMHS ensuring they work to provide GPs with a service framework that facilitates clinical decision-making.

The third challenge identified in this study was that of satisfactory ongoing communication between the GP and the different providers of care. The findings suggest that these GPs have generally good links with their local CAMHS and health visitors, but there are mixed feelings regarding the voluntary sector, and overall poor communication links with social services. Personal relationships and contact with CAMH service staff help gain clarity about the types of referrals that can be accepted, but the absence of this relationship in some cases makes it very difficult to know whether to refer. The care pathways developed locally have not helped in making this communication easier and give the clear impression that they were not intended to inform GPs in everyday practice. These findings point to the need for better collaboration between CAMHS and primary care workers in the development and implementation of care pathways. The findings also highlight longstanding structural weaknesses in the services for children in general, with agencies with different financial, legal and professional responsibilities struggling to develop coherent delivery of mental health and social care services at the primary care level. The absence of a well-described set of clinical and care 'products' that could be available to every practitioner and primary care service is a serious drawback to future delivery of evidence-based services in this field. This could be accomplished if NHS and social services were able to align their service frameworks and conceptually bring together the differing approaches used to conduct a social and clinical assessment. The development of an initiative such as IAPT (Improving Access to Psychological Therapies; http://www.iapt.nhs.uk/) for children and young people may be one way of managing the care pathway more effectively. Service provision of this type, however, must be accompanied by information on how it should be used, by whom, and for what purposes, as already noted for primary care services recognising and managing depression in adults. Whatever measure is adopted, having a range of health and social care products, clearly marked, and extending over the care pathway for CAMHS, would give GPs much greater clarity and signposting than what is currently available.

\section{CONCLUSIONS}

This is a pilot study with the objective of investigating GP perceptions and experiences in the referral of mentally ill and behaviourally disturbed children and adolescents. The quantitative analysis of the Cambridge and Peterborough Foundation Trust data showed that referrals of children and adolescents with potential mental health problems originating from GPs were three times more likely to end in a rejection by CAMHS compared to other referrers. The qualitative results confirm previous studies that detecting the signs and symptoms of mental illness in young people is a challenge for GPs. There appears to be no standardised specialised knowledge base from which GPs can develop their clinical decision-making skills for child and adolescent mental health. The hypotheses generated are that the absence of management tools to aid GPs in their mental health assessment is a causal factor in the higher rejection rate by CAMHS further down the pathway, and their low knowledge levels about child and adolescent mental health is a driver for poor decision-making in the referral process. The findings also highlight the need for 
continuing professional education for GPs in the area of common child and adolescent mental health problems to improve their clinical assessment and decisionmaking. Overall, and despite these difficulties, GPs have generally good links with their local CAMHS and health visitors. In contrast, there appear to be modest to poor links with the voluntary sector and social services. There are longstanding structural weaknesses in the services for children and young people in general, reflected in poor multiagency co-operation at the primary care level. GP-friendly guidelines are urgently required that will aid decision-making, help with understanding the referrals process, and most importantly, operate in the best interests of the mentally ill child and family. We look to managers of both commissioning and providing organisations, as well as future research, to drive forward the development of tools, protocols and health service structures to help aid the efficiency and effectiveness of recognising and treating mental illness in young people.

\section{Author affiliations}

${ }^{1}$ Department of Engineering, Engineering Design Centre, University of Cambridge, Cambridge, UK

${ }^{2}$ Department of Psychiatry, Developmental Psychiatry, University of Cambridge, Cambridge, UK

${ }^{3}$ Cambridge and Peterborough NHS Foundation Trust, Cambridge, UK

Contributors (SH, MO, IG, VD) contributed to the design of the study and the writing of the article. SH conducted the interviews and analysis. MO conducted the quantitative analysis.

Funding This work was supported by the National Institute for Health Research Collaborations for Leadership in Applied Health Research and Care (CLAHRC), grant number RNAG-186. The opinions expressed are those of the authors, not the funders.

Competing interests None.

Provenance and peer review Not commissioned; externally peer reviewed.

Data sharing statement No additional data are available.

\section{REFERENCES}

1. Ford T, Goodman R, Meltzer H. The British Child and Adolescent Mental Health Survey 1999: the prevalence of DSM-IV disorders. J Am Acad Child Adolesc Psychiatry 2003;42:1203-11.

2. Green F, McGinnity A, Metlzer H, et al. Mental health of children and young people in Great Britain, 2004. Office for National Statistics, 2005; Hampshire, UK: Palgrave Macmillan. ISBN 1-4039-8637-1.

3. Meltzer $\mathrm{H}$, Ford $\mathrm{T}$, Goodman $\mathrm{R}$, et al. The burden of caring for children with emotional or conduct disorders. Int J Family Med 2011; doi:10.1155/2011/801203.

4. Scott S, Knapp M, Henderson J, et al. Financial cost of social exclusion: follow up study of antisocial children into adulthood. BMJ 2001;323:191.

5. Kessler RC, Heeringa S, Lakoma MD, et al. Individual and societal effects of mental disorders on earnings in the USA: results from the National Comorbidity Survey Replication. Am J Psychiatry 2008;165:703-711.

6. World Health Organisation. Caring for children and adolescent with mental disorders: setting WHO directions. 2003; ISBN 924159063 7. http://www.who.int/mental_health/media/en/785.pdf (accessed 6 Sept 2012).

7. De Bleser L, Depreitere R, De Waele K, et al. Defining pathways J Nurs Manag 2006;14:553-63.

8. World Health Organisation. Atlas: child and adolescent mental health resources: global concerns, implications for the future. 2005; ISBN 924156304 4. Available at: http://www.who.int/mental_health/ resources/Child ado atlas.pdf (accessed 6 Sept 2012).
9. World Health Organisation. Advocacy for mental health. (Mental health policy and service guidance package). 2003; ISBN 924 154590 9. Available at: http://www.who.int/mental_health/resources/ en/Advocacy.pdf (accessed 6 Sept 2012).

10. Department of Health. Mental Health Policy Implementation Guidea learning and development toolkit. 2007. Available at: http://www. dh.gov.uk/prod_consum_dh/groups/dh_digitalassets/@dh/@en/ documents/digitalasset/dh_073681.pdf (accessed 6 Sept 2012).

11. Department of Health. National service framework for children, young people and maternity services: the mental health and psychological wellbeing of children and young people. 2004. Available at: http://www.dh.gov.uk/en/Publicationsandstatistics/ Publications/PublicationsPolicyAndGuidance/DH_4089114 (accessed September 6, 2012).

12. Cambridgeshire and Peterborough NHS Foundation Trust. A guide to mental health care pathways. 2010.

13. Kazak AE, Hoagwood K, Weisz JR, et al. A meta-systems approach to evidence-based practice for children and adolescents. Am Psychol 2010;65:85-97.

14. Evans-Lacko $S$, Jarrett $M$, McCrone $P$, et al. Facilitators and barriers to implementing clinical care pathways. BMC Health Serv Res 2010;10:182.

15. Jones A. Perceptions on the standardization of psychiatric work: development of a care pathway. J Psychiatr Ment Health Nurs 2004;11:705-13.

16. de Luc K. Care pathways: an evaluation of their effectiveness. $J$ Adv Nurs 2000;32:485-96

17. Kent $\mathrm{P}$, Chalmers $\mathrm{Y}$. A decade on: has the use of integrated care pathways made a difference in Lanarkshire? J Nurs Manag 2006;14:508-20.

18. Sayal K, Taylor E. Detection of child mental health disorders by general practitioners. Br J Gen Pract 2004;54:348-352.

19. Sayal K. Pathways to care for children with mental health problems. J Child Psychol Psychiatry 2006;47:649-59.

20. Sayal K, Amarasinghe M, Robotham S, et al. Quality standards for child and adolescent mental health in primary care. BMC Fam Pract 2012;13:51.

21. Sayal K, Tischler V, Coope C, et al. Parental help-seeking in primary care for child and adolescent mental health concerns: qualitative study. Br J Psychiatry 2010;197:476-81.

22. Office of National Statistics. Available at: http://www.ons.gov.uk/ons/ $\mathrm{rel} / \mathrm{census} / \mathrm{census}-2001-\mathrm{key}$-statistics/

local-authorities-in-england-and-wales/index.html. (accessed September 6, 2012).

23. Tong A, Sainsbury P, Craig J. Consolidated criteria for reporting qualitative research (COREQ): a 32-item checklist for interviews and focus groups. Int J Qual Health Care 2007;19:349-57.

24. Crilly N, Blackwell A, Clarkson P. Graphic elicitation: using research diagrams as interview stimuli. Qual Res 2006;6:341-66.

25. Umoquit M, Dobrow M, Lemieux-Charles L, et al. The efficiency and effectiveness of utilizing diagrams in interviews: an assessment of participatory diagramming and graphic elicitation. BMC Med Res Methodol 2008;8:53.

26. Miles M, Huberman A. Qualitative data analysis: an expanded sourcebook. 2nd edn. Sage 1994;50-88.

27. World Health Organisation. International Statistical Classification of Diseases and Health Related Problems, Geneva, 2004.

28. Niranjan A, Corujo A, Ziegelstein RC, et al. Depression and heart disease in US adults. Gen Hosp Psychiatry 2012;34:254-61.

29. National Institute for Health and Clinical Excellence. Depression in children and young people: identification and management in primary, community, and secondary care. Clinical guideline 28. Available at: http:// publications.nice.org.uk/depression-in-children-and-young-people-cg28 (accessed 21 May 2012).

30. American Psychiatric Association. Diagnostic and statistical manual of mental disorders: DSM-IV-TR. 4th edn.

31. Moran P, Coffey $\mathrm{C}$, Romaniuk $\mathrm{H}$, et al. The natural history of self-harm from adolescence to young adulthood: a population-based cohort study. Lancet 2012;379:236-43.

32. Wilkinson $\mathrm{P}$, Kelvin R, Roberts $\mathrm{C}$, et al. Clinical and psychosocial predictors of suicide attempts and nonsuicidal self-injury in the Adolescent Depression Antidepressants and Psychotherapy Trial (ADAPT). Am J Psychiatry 2011;168:495-501.

33. Vila M, Kramer T, Obiols JE, et al. Adolescents who are frequent attenders to primary care: contribution of psychosocial factors. Soc Psychiatry Psychiatr Epidemiol 2012;47:323-9.

34. Kramer T, Iliffe S, Gledhill J, et al. Recognising and responding to adolescent depression in general practice: developing and implementing the Therapeutic Identification of Depression in Young people (TIDY) programme. Clin Child Psychol Psychiatry 2012;17:482-94. 
35. Merry SN, Stasiak K, Shepherd M, et al. The effectiveness of SPARX, a computerised self help intervention for adolescents seeking help for depression: randomised controlled non-inferiority trial. BMJ 2012;344:e2598.

36. Iliffe S, Gallant C, Kramer T, et al. Therapeutic identification of depression in young people: lessons from the introduction of a new technique in general practice. Br J Gen Pract 2012;62:e174-82.

37. Pfaff JJ, Acres JG, McKelvey RS. Training general practitioners to recognise and respond to psychological distress and suicidal ideation in young people. Med J Aust 2001;174:222-6.

38. Steele M, Zayed R, Davidson B, et al. Referral patterns and training needs in psychiatry among primary care physicians in canadian rural/remote areas. J Can Acad Child Adolesc Psychiatry 2012;21:111-23.
39. Lester $\mathrm{H}$, Khan $\mathrm{N}$, Jones $\mathrm{P}$, et al. Service users' views of moving on from early intervention services for psychosis: a longitudinal qualitative study in primary care. Br J Gen Pract 2012;62:e183-90.

40. Younes N, Gasquet I, Gaudebout P, et al. General practitioners' opinions on their practice in mental health and their collaboration with mental health professionals. BMC Fam Pract 2005;6:18.

41. Piek E, Nolen WA, van der Meer K, et al. Determinants of (non-) recognition of depression by general practitioners: results of the Netherlands Study of Depression and Anxiety. J Affect Disord 2012;138:397-404.

42. Connolly A, Gaehl E, Martin $\mathrm{H}$, et al. Underdiagnosis of dementia in primary care: variations in the observed prevalence and comparisons to the expected prevalence. Aging Ment Health 2011;15:978-84. 